\title{
The Quest for a Truly Parsimonious Airfoil Parameterization Scheme
}

\author{
András Sóbester*, Thomas Barrett ${ }^{\dagger}$ \\ University of Southampton, Southampton, Hampshire, SO17 1BJ, UK
}

\begin{abstract}
The conceptual phase of the aircraft design process demands a parsimonious description of the airframe geometry. While there is no hard and fast upper limit on the affordable number of variables, the so-called 'curse of dimensionality' must always be kept in mind: if a thorough, conceptual level search of a one-variable space can be accomplished by the evaluation of $n$ candidate designs, the same level of thoroughness (however one chooses to define this) will demand $n^{k}$ evaluations in $k$-dimensional space. Therefore, to make the design search tractable and the complexity of the geometry definition at a level where the effect of the variables can be readily understood by the designer, the number of airframe definition variables should be minimized. This has an impact on all of the components, perhaps most importantly on airfoiltype section definitions that feature on all 'wing-like' surfaces. It is therefore imperative to define these sections as parsimoniously as possible. In this paper we examine a series of parameterization schemes, whose chief conception criterion was conciseness in terms of the number of design variables. Another constraint we are considering is the ease of implementation in a commercial off-the-shelf Computer Aided Design engine.
\end{abstract}

\section{Introduction}

$\mathbf{T}$ HE 'curse of dimensionality', that is, the observation that the size of a $k$-dimensional space observed at $q$ levels is $O\left(q^{k}\right)$, presents conceptual designers with a significant challenge in terms of reducing the number of design variables. This is especially pressing when it comes to defining airfoil sections, as these feature on every lifting surface on an airframe and therefore are often responsible for a significant fraction of the overall number of design variables. This paper presents a number of potential airfoil representation formulations designed with low dimensionality as the chief driver.

The search for practically useful parametric airfoils is almost as old as heavier than air flight itself. A plethora of airfoil geometry definition schemes have been proposed over the years and many different criteria could be used to arrange these into a taxonomy. Here, in a brief overview of existing approaches, we choose to simply position them along a continuum of design variable scopes. In other words, we classify them according to the geometrical extent (in terms of the overall size of the airfoil) of the influence of any particular design variable, as this criterion feels the most germane when it comes to clarifying the place of our proposed schemes in the rather broad context of airfoil description methodology.

\footnotetext{
${ }^{*}$ Lecturer, Computational Engineering and Design Group, AIAA member.

${ }^{\dagger}$ AIAA Member
} 
Consider the extremes of this scale. If a computational fluid dynamics mesh is generated around a putative airfoil, one could designate the coordinates of the mesh points falling onto the airfoil surface as design variables. Sometimes combined with a related mapping of the rest of the mesh, as well as a smoothness constraint designed to prevent the emergence of 'jagged' airfoils, this is a highly successful scheme when a local improvement is sought in relation to the starting point of the design process. The search can be particularly efficient when adjoint gradients are available at a computational cost that is independent of problem dimensionality. A classic example is the control theory based formulation of Jameson. ${ }^{1}$ Clearly, in this case the influence of a design variable is restricted to the immediate vicinity of its corresponding mesh point.

Staying with Jameson's work, one can also find here an example of the opposite end of the scale, where a design parameter's scope is the entire airfoil. In Ref. 2 he describes the solution of the potential flow equations over an airfoil, which results from a circle, via a complex conformal mapping (a mapping, which is, incidentally, also applied to the mesh). A very similar example is that of the well-known Jukowski airfoil - in both cases the impact of changes to the coordinates of the circle being mapped can be seen across the entire airfoil.

All other methods populate the span between these two extremes. Indeed, some can take up different locations along this continuum, depending on how the designer chooses to implement them. For example, the 'bump' functions, introduced by Hicks and Henne ${ }^{3}$ at the dawn of the computeraided design age, can be added to the baseline airfoil scaled to the entire chord, or, more locally, to a limited part thereof (see Ref. 4 for an 18-variable example). Another way of controlling the scope of the Hicks-Henne parameterisation method is to restrict the range of permitted chordwise movement of the peak of the bump (while the base of the bump rests on the full chord).

Also, to some extent, variable in scope, is the scheme described recently by Kulfan. ${ }^{5}$ At the heart of her airfoil description lies a generic template, containing a square root term (to ensure a round leading edge), and a ' $1-\mathrm{x} /$ chord' term to close the trailing edge (with another term specifying a finite trailing-edge thickness). The actual parameterisation is encapsulated by a shape function term with variable flexibility. At its stiffest, the shape function can depend on a single variable - in this case we can control the nose radius on both the lower and upper surfaces at the same time. It is also possible to set up a one variable shape function to control the boat-tail angle. Both of these choices place us about half-way down the scope continuum, as the impact of any change affects either the fore or the aft section of the airfoil. More flexible shape functions can come, for example, in the form of a Bernstein-polynomial partition of unity, which gives increasingly finer and, in some sense, more localized control of the shape (as an illustration of the flexibility of the shape function, Ref. 5 includes $8^{\text {th }}$ order Bernstein polynomial approximations of the RAE2822 airfoil, which take the approximation errors well below wind tunnel model tolerances).

Let us now consider a few examples of formulations with more specific places on the parameter scope scale. Lépine et al. ${ }^{6}$ and Painchaud-Ouellet et al. ${ }^{7}$ use NURBS curves to define their airfoils, where the coordinates and the weights of the control points are the design variables. The significant impact of each is, of course, restricted to the vicinity of the relevant control point and therefore this scheme is close to the local end of the scope scale. 
$6^{\text {th }}$ order polynomials form the basis of the PARSEC formulation. ${ }^{8}$ Each of the 11 design variables is connected to a very specific feature of the airfoil: leading edge radius, upper and lower crest location, curvatures at the crests, trailing edge ordinates, thickness, tail direction and wedge angle. This is, therefore also fairly near to the local end of the scale: each variable affects a well-defined section of the airfoil.

Near the global end we find the classic 4-digit NACA foils. The variables here refer to specific features of the airfoil (max thickness and its location and maximum camber), but, each of these refers to both the upper and the lower surface of the airfoil and thus any change in any of the design variables has some impact on every point. Also at the global end we find the orthogonal basis functions of Robinson and Keane. ${ }^{9}$ These functions represent features extracted from the NASA Phase 2 supercritical airfoils (described by Harris ${ }^{10}$ ) and the variables, which are the weights of these bases, affect the shape of the entire airfoil.

As we stated at the outset, our main aim here is to consider a number of parsimonious parametric airfoils, that is, section descriptions that can be controlled through a relatively small number of parameters (accepting inevitable sacrifices in terms of flexibility, i.e., the ability of the parametric airfoil to take up a wide range of shapes).

It is clear that, in terms of the above classification, parametric airfoils suitable for conceptual design cannot be at the local end of the parameter scope scale. We therefore start, in what follows, around the mid-point of the scale, with three schemes that, as we shall see, share a number of features. The simplest of this trio is based on cubic splines (Section II) - this is followed by two B-spline based schemes (Sections III and IV). We then tackle the global extreme of the scale, via a variable dimensionality formulation based on defining new airfoils in terms of the features of existing, 'classic' sections (Section V).

\section{A Parametric Airfoil Based on Cubic Splines}

We begin with a six-variable formulation based on a pair of cubic splines. The main advantage of this scheme lies in its simplicity: setup times are minimal in most programming environments and the mathematical definition is also simpler than that of the B-spline-based schemes we shall tackle later on. For these reasons we have found this a rather effective teaching tool. The main drawback is that it is only suitable for low speed applications - a pair of splines controlled from their endpoints (which is how we shall construct this parametric section) do not have the flexibility required to generate the near-flat surface regions found on supercritical airfoils. In what follows we briefly outline the mathematical formulation of this airfoil definition method.

\section{Ferguson's Parametric Splines}

The specific spline formulation that forms our starting point is that of The Boeing Company's James Ferguson, whose seminal 1964 paper ${ }^{11}$ lies at the foundation of the curve (and surface) definition algorithms built into modern Computer Aided Design (CAD) tools. Although Bèzier and others have subsequently published slightly different and now better known forms of cubics, their basic logic is very similar to what we now proceed to describe. 


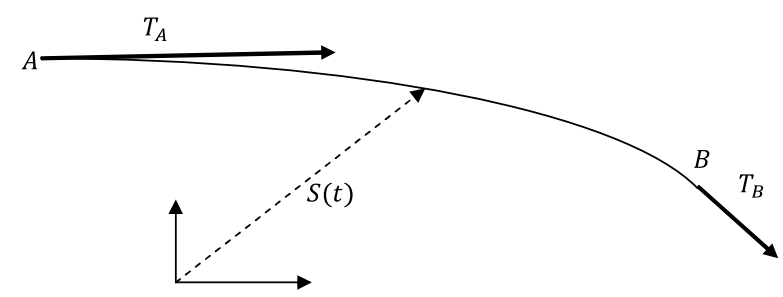

Fig. 1 Ferguson spline and its boundary conditions.

We seek a parametric curve $\mathbf{S}(t)$ with $t \in[0,1]$, connecting two points $\mathbf{S}(0)=\mathbf{A}$ and $\mathbf{S}(1)=\mathbf{B}$. We impose two tangents on the curve: $\mathrm{d} \mathbf{S} /\left.\mathrm{d} t\right|_{t=0}=\mathbf{T}_{A}$ and $\mathrm{d} \mathbf{S} /\left.\mathrm{d} t\right|_{t=1}=\mathbf{T}_{B}$, as shown in Figure 1. We define the curve as the polynomial

$$
\mathbf{S}(t)=\sum_{i=0}^{3} \mathbf{a}_{i} t^{i}, t \in[0,1]
$$

We find the four vectors required to define the curve by setting the endpoint conditions:

$$
\begin{aligned}
& \mathbf{A}=\mathbf{a}_{0} \\
& \mathbf{B}=\mathbf{a}_{0}+\mathbf{a}_{1}+\mathbf{a}_{2}+\mathbf{a}_{3} \\
& \mathbf{T}_{A}=\mathbf{a}_{1} \\
& \mathbf{T}_{B}=\mathbf{a}_{1}+2 \mathbf{a}_{2}+3 \mathbf{a}_{3}
\end{aligned}
$$

and by re-arranging:

$$
\begin{aligned}
& \mathbf{a}_{0}=\mathbf{A} \\
& \mathbf{a}_{1}=\mathbf{T}_{A} \\
& \mathbf{a}_{2}=3[\mathbf{B}-\mathbf{A}]-2 \mathbf{T}_{A}-\mathbf{T}_{A} \\
& \mathbf{a}_{3}=2[\mathbf{A}-\mathbf{B}]+\mathbf{T}_{A}+\mathbf{T}_{B} .
\end{aligned}
$$

Substituting them back into Equation (1) we obtain:

$$
\mathbf{S}(t)=\mathbf{A}\left(1-3 t^{2}+2 t^{3}\right)+\mathbf{B}\left(3 t^{2}-2 t^{3}\right)+\mathbf{T}_{A}\left(t-2 t^{2}+t^{3}\right)+\mathbf{T}_{B}\left(-t^{2}+t^{3}\right),
$$

or in matrix form:

$$
\mathbf{S}(t)=\left[\begin{array}{llll}
1 & t & t^{2} & t^{3}
\end{array}\right]\left[\begin{array}{cccc}
1 & 0 & 0 & 0 \\
0 & 0 & 1 & 0 \\
-3 & 3 & -2 & -1 \\
2 & -2 & 1 & 1
\end{array}\right]\left[\begin{array}{c}
\mathbf{A} \\
\mathbf{B} \\
\mathbf{T}_{A} \\
\mathbf{T}_{B}
\end{array}\right]
$$

$\mathbf{S}(t)$ is, essentially, a Hermitian interpolant and the bracketed factors in equation (4) can be viewed as its basis functions - Figure 2 conveys an intuitive understanding of their effect on the shape of the interpolant. We now proceed to discuss the suggested airfoil parameterization scheme based on the formulation outlined in the introduction. 


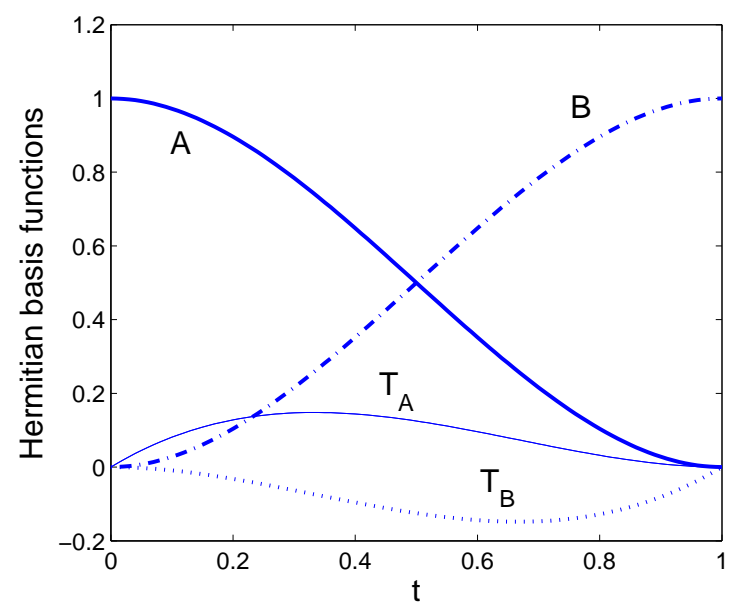

Fig. 2 The four basis functions of equation (4) shown with their respective multipliers.

\section{Constructing the Parametric Airfoil}

The geometry is illustrated in Figure 3. The tangent of the upper surface $\mathbf{S}^{u}(t)$ in $\mathbf{A}$ (at the leading edge) is denoted by $\mathbf{T}_{A}^{\text {upper }}$ and its tangent in $\mathbf{B}$ by $\mathbf{T}_{B}^{\text {upper }}$ - the same logic is used for the nomenclature of the lower surface.

The shape of the airfoil is thus defined by the orientation and the magnitude of the tangent vectors. $\mathbf{T}_{A}^{\text {upper }}$ and $\mathbf{T}_{A}^{\text {lower }}$ will always be pointing vertically downwards and upwards respectively, with their magnitude defining the tension in the spline, thus controlling the 'bluntness' of the leading edge. $\alpha_{c}$, which might be called the camber angle, defines the orientation of $\mathbf{T}_{B}^{\text {lower }}$, while the boattail angle $\alpha_{b}$ determines the orientation of $\mathbf{T}_{B}^{\text {upper }}$. The magnitudes (tangent tensions) of these vectors determine the shape of the middle section of the airfoil.

It can be seen that, in addition to having relatively few parameters (six), these also relate intuitively to geometrical features of the airfoil and, to some extent, even to its aerodynamic features: the tensions in the tangents at $\mathbf{A}$ determine the angle of attack capability, the tangents at $\mathbf{B}$ are related to the pressure drag and drag rise characteristics and $\alpha_{c}$ has a major influence on $c_{l}$. Structurally, the boattail angle $\alpha_{b}$ will, together with the nose tangent magnitudes, control the cross-sectional area of the wing.

We conclude our discussion of the fundamentals of this airfoil definition scheme with a note on finite thickness trailing edges. Should such an airfoil be required, the curves $\mathbf{S}^{u}(t)$ and $\mathbf{S}^{l}(t)$ (see Figure 3) will not meet in $\mathbf{B}$, but will have separate endpoints on a perpendicular raised to the chord in $\mathbf{B}$, at ordinates $\epsilon_{u}$ and $\epsilon_{l}$ respectively. This requires no change to the basic formulation, other than replacing $\mathbf{B}(1,0)$ with $\mathbf{B}^{u}\left(1, \epsilon_{u}\right)$ and $\mathbf{B}^{l}\left(1, \epsilon_{l}\right)$ in the definitions of the two surfaces of the airfoil. The ordinates $\epsilon_{u}$ and $\epsilon_{l}$ could be fixed at certain values, but can also be allowed to function as additional design variables. If this is deemed necessary in an optimization context, a seventh design variable can be added, say, $\epsilon$, where the the top and bottom surfaces are anchored at the trailing edge by $\mathbf{B}^{u}(1, \epsilon / 2)$ and $\mathbf{B}^{l}(1,-\epsilon / 2)$ respectively. 


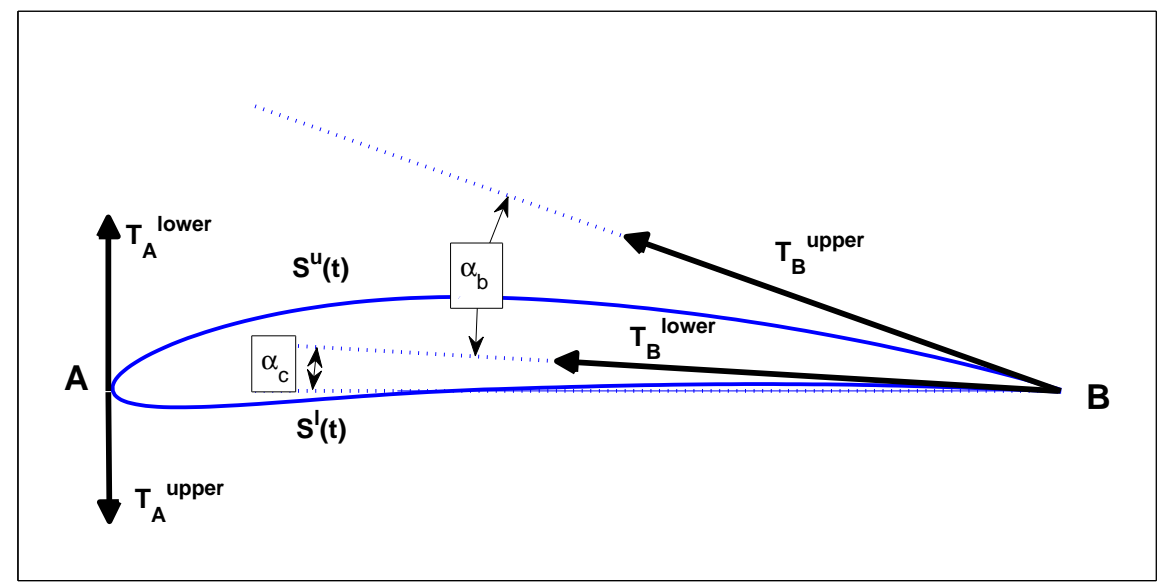

Fig. 3 The airfoil parameterization scheme based on two Ferguson splines. The parametric curves $\mathbf{S}^{u}(t)$ and $\mathbf{S}^{l}(t)$ describe the upper and lower surfaces respectively.

As we indicated at the outset, the flexibility of this parametric airfoil may be too limited to recreate certain classes of high speed airfoils. Nevertheless, there are numerous other 'classic' shapes that this simple and robust formulation can approximate rather closely. Such approximations can be obtained by minimizing some difference metric (e.g., mean squared error) between the parametric airfoil and the target of the approximation, subject to the design variables $\left|\mathbf{T}_{A}^{\text {upper }}\right|,\left|\mathbf{T}_{A}^{\text {lower }}\right|$, etc. Here are two examples of parameter sets obtained in this way:

\begin{tabular}{|c|c|c|c|c|c|c|c|c|}
\hline Target airfoil & $\left|\mathbf{T}_{A}^{\text {upper }}\right|$ & $\left|\mathbf{T}_{A}^{\text {lower }}\right|$ & $\left|\mathbf{T}_{B}^{\text {upper }}\right|$ & $\left|\mathbf{T}_{B}^{\text {lower }}\right|$ & $\alpha_{c}[\mathrm{deg}]$ & $\alpha_{b}[\mathrm{deg}]$ & $\epsilon_{l}$ & $\epsilon_{u}$ \\
\hline NACA 5410 & 0.1584 & 0.1565 & 2.1241 & 1.8255 & 3.8270 & 11.6983 & -0.0032 & 0.0012 \\
\hline NACA 21012 & 0.1674 & 0.2402 & 2.2482 & 1.3236 & -8.7800 & 17.2397 & -0.0074 & -0.0080 \\
\hline
\end{tabular}

The reader interested in more such reconstructions, implementation details, flexibility analysis and a multi-objective design study based on this parametric airfoil, will find these in Ref. 12 . We now move on to consider another simple parameterisation scheme of the same dimensionality $(k=6)$, this time using a set of interpolation points and a more sophisticated geometrical formulation: a B-spline.

\section{A B-Spline Based Parametric Airfoil}

\section{Constructing an Interpolating B-Spline}

Let the following vector curve define a $p$-th order B-spline:

$$
\mathbf{S}(t)=\sum_{i=0}^{n} N_{i, p}(t) \mathbf{P}_{i}
$$

6

Submitted to the American Institute of Aeronautics and Astronautics 
for $t \in[0,1] . P_{i}$ are the $(n+1)$ control points, and $N_{i, p}(t)$ are the $p$-th degree B-spline basis functions. The knot vector, $\mathbf{T}$, containing $(m+1)$ knots, is defined as

$$
\mathbf{T}=\{\underbrace{0, \ldots, 0}_{p+1}, t_{p+1}, \ldots, t_{m-p-1}, \underbrace{1, \ldots, 1}_{p+1}\}
$$

in which $m=n+p+1$. The B-spline basis functions of degree $p$ (order $p+1$ ) are defined as

$$
N_{i, p}(t)=\frac{t-t_{i}}{t_{i+p}-t_{i}} N_{i, p-1}(t)+\frac{t_{i+p+1}-t}{t_{i+p+1}-t_{i+1}} N_{i+1, p-1}(t) .
$$

Note that this linear combination of lower order basis functions is defined everywhere, though we do not actually need it outside the range defined by the extremities of the knot vector. This recursive formulation (due to $\mathrm{Cox}^{13}$ and De Boor ${ }^{14}$ ) can be pictured intuitively as a triangle, whose apex is the basis function to be calculated, the two basis functions needed for its calculation are right underneath it and so on. The base of the triangle, which effectively controls the ranges of influence of the control points, is defined as:

$$
N_{i, 0}(t)=\left\{\begin{array}{cc}
1 & \text { if } \quad t_{i} \leq t<t_{i+1} \\
0 & \text { otherwise }
\end{array}\right.
$$

When evaluating the curve $\mathbf{S}(t)$, for each $t$ one finds the knot span in which it lies, computes the relevant basis functions, and multiplies these by the corresponding control points using Equation (6).

Let us now consider interpolation using a B-spline thus defined, as we will need this for the actual definition of the airfoil. More specifically, we need to calculate the control point locations that cause the B-spline to interpolate a set of specified points in a given order. This will make the scheme easier to implement in CAD packages that only allow the specification of interpolation points (and do not allow direct editing of control points). If the $(n+1)$ data points to be interpolated are each denoted by $\mathbf{D}_{l}$, let these correspond to parameter values $t_{l}$. Then Equation (6) can be used to write the interpolation condition

$$
\mathbf{D}_{l}=\mathbf{S}\left(t_{l}\right)=\sum_{i=0}^{n} N_{i, p}\left(t_{l}\right) \mathbf{P}_{i}, \quad 0 \leq l \leq n
$$

Here, the basis functions $N_{i, p}\left(t_{l}\right)$ collectively form a $(n+1) \times(n+1)$ matrix, $\mathbf{N} . \mathbf{D}_{l}$ and $\mathbf{P}_{i}$ are both vectors in 2-dimensional space, and are rows of the $(n+1) \times 2$ matrices $\mathbf{D}$ and $\mathbf{P}$. The above relation can therefore be written as the linear system

$$
\mathbf{D}=\mathbf{N P}
$$

which is solved for the $n+1$ control points stored in $\mathbf{P}$. The corresponding curve can then be calculated by substitution into Equation (6).

As we shall see shortly, a final operation needed for the specification of our parametric airfoil is to specify the start and end gradients of the B-spline curve. We do this following Piegl and Tiller. ${ }^{15}$ A new knot is inserted into the first and last knot spans by averaging the $t_{l}$ 's. The matrix of basis functions is calculated as normal using the new knot vector, and subsequently a new matrix $\mathbf{N}$ is 


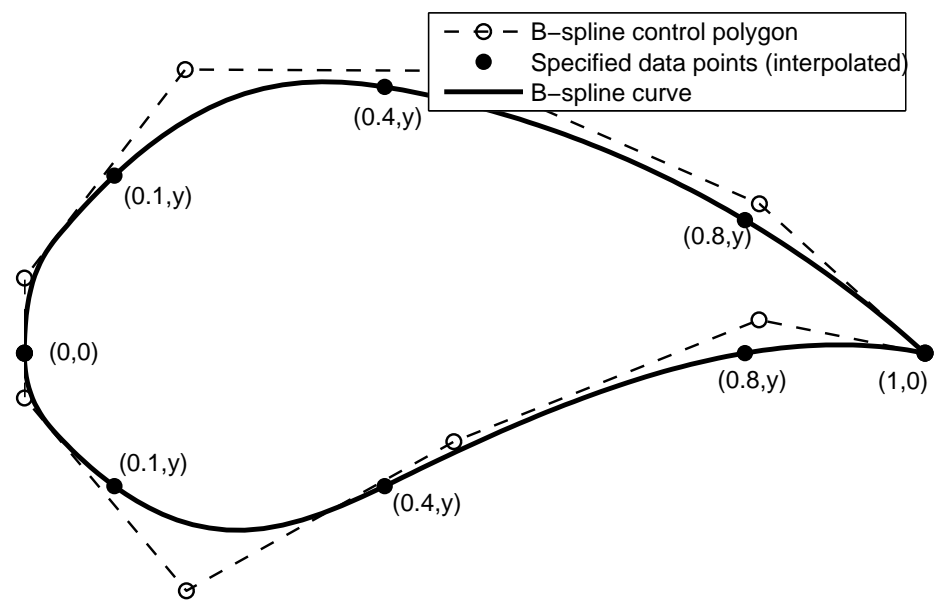

Fig. 4 A B-Spline based six-variable parameterization of an airfoil shape.

formed by inserting the B-spline basis function derivatives at the start and end points into the second and penultimate rows of $\mathbf{N}$, respectively. The following linear system is formed

$$
\left[\begin{array}{c}
\mathbf{D}_{0} \\
\mathbf{D}_{0}^{\prime} \\
\mathbf{D}_{1} \\
\cdots \\
\mathbf{D}_{n-1} \\
\mathbf{D}_{n}^{\prime} \\
\mathbf{D}_{n}
\end{array}\right]=\mathbf{N}\left[\begin{array}{c}
\mathbf{P}_{0} \\
\mathbf{P}_{1} \\
\cdots \\
\mathbf{P}_{m-1} \\
\mathbf{P}_{m}
\end{array}\right]
$$

where $\mathbf{D}_{0}^{\prime}$ and $\mathbf{D}_{n}^{\prime}$ are the specified first derivatives of the B-spline at its start and end points, respectively. This system is solved for the $m+1 \mathrm{~B}$-spline control points and the corresponding curve is once again defined by Equation (6). Note that $m=n+2$, because the specified start and end derivatives increase the number of B-spline control points.

\section{Airfoil Definition With B-splines}

We define the parametric, airfoil through two cubic B-splines, one for the upper surface $\left(\mathbf{S}^{u}\right)$ and one for the lower surface $\left(\mathbf{S}^{l}\right)$. These are specified through two requirements. The first is that the curves interpolate a set of points with fixed ordinates. The second is that they have specified gradients at the leading edge - see Figure 4.

The abscissas of the data points are fixed at $x / c=\{0,0.1,0.4,0.8,1\}$, where $c$ denotes the airfoil chord, and the leading and trailing edge positions are fixed. The ordinates of the three points on each surface are the profile design variables, giving a total of six design variables for this parameterisation (considering a sharp trailing edge). The trailing edge gradients are left to float. The leading edge gradients are fixed at $\mathrm{d} \mathbf{S}^{u} /\left.\mathrm{d} t\right|_{t=0}=[0,0.5]$ for the upper surface, and $\mathrm{d} \mathbf{S}^{l} /\left.\mathrm{d} t\right|_{t=0}=[0,-0.3]$ on the lower surface. These are values that, along with those of of the interpolation point abscissas we have chosen, appear to work well for a wide range of airfoil shapes. 


\section{B-Splines Without Interpolation Points}

In Section II we have looked at the possibility of controlling a cubic spline from its end points to an extent sufficient to produce airfoil shapes. We shall now repeat the exercise - this time by modifying the B-Spline based formulation introduced above.

The first thing we need to alter is the knot vector, which will now be:

$$
\mathbf{T}=\{0,0,0,0,1,1,1,1\}
$$

giving a cubic B-spline where the curve is forced to be tangent to the control polygon at its extremities. Next, we rewrite the matrix $\mathbf{D}$ for the upper surface $\left(\mathbf{D}^{u}\right)$ and the lower surface $\left(\mathbf{D}^{l}\right)$ to match the setup and the notation developed for the Ferguson spline formulation (Figure 3):

$$
\begin{gathered}
\mathbf{D}^{u}=\left[\begin{array}{c}
\mathbf{D}_{0}^{u} \\
\mathbf{D}_{0}^{\prime u} \\
\mathbf{D}_{1}^{\prime u} \\
\mathbf{D}_{1}^{u}
\end{array}\right]=\left[\begin{array}{cc}
0 & 0 \\
0 & \left|\mathbf{T}_{A}^{\text {upper }}\right| \\
\left|\mathbf{T}_{B}^{\text {upper }}\right| \cos \left(\alpha_{b}+\alpha_{c}\right) & \left|\mathbf{T}_{B}^{\text {upper }}\right| \sin \left(\alpha_{b}+\alpha_{c}\right) \\
0 & 1
\end{array}\right], \\
\mathbf{D}^{l}=\left[\begin{array}{c}
\mathbf{D}_{0}^{l} \\
\mathbf{D}_{0}^{\prime l} \\
\mathbf{D}_{1}^{\prime l} \\
\mathbf{D}_{1}^{l}
\end{array}\right]=\left[\begin{array}{cc}
0 & 0 \\
0 & \left|\mathbf{T}_{A}^{\text {lower }}\right| \\
\left|\mathbf{T}_{B}^{\text {lower }}\right| \cos \alpha_{c} & \left|\mathbf{T}_{B}^{\text {lower }}\right| \sin \alpha_{c} \\
0 & 1
\end{array}\right]
\end{gathered}
$$

The rest of the calculation procedure is the same as described in Section III; the B-spline basis function derivatives are inserted as the second and penultimate rows of $\mathbf{N}$ and the system $\mathbf{D}=\mathbf{N P}$ (written twice, once for each surface) is solved for the control points $\mathbf{P}$ (once again, we recommend the text by Piegl and Tiller, ${ }^{15}$ this time as a good source of additional detail on setting up B-splines with specified end-derivatives).

Approximating once again the two NACA airfoils we considered in the section about the simple Ferguson spline airfoil formulation, we find that the design variable values found for this formulation are, unsurprisingly, very similar:

\begin{tabular}{|c|c|c|c|c|c|c|c|c|}
\hline Target airfoil & $\left|\mathbf{T}_{A}^{\text {upper }}\right|$ & $\left|\mathbf{T}_{A}^{\text {lower }}\right|$ & $\left|\mathbf{T}_{B}^{\text {upper }}\right|$ & $\left|\mathbf{T}_{B}^{\text {lower }}\right|$ & $\alpha_{c}[\mathrm{deg}]$ & $\alpha_{b}[\mathrm{deg}]$ & $\epsilon_{l}$ & $\epsilon_{u}$ \\
\hline NACA 5410 & 0.1357 & 0.1559 & 2.2752 & 1.8451 & 3.7141 & 10.5696 & -0.0030 & 0.0046 \\
\hline NACA 21012 & 0.1475 & 0.2693 & 2.4167 & 0.9983 & -9.4204 & 17.3817 & -0.0084 & -0.0055 \\
\hline
\end{tabular}

\section{Linear Combinations of Basis Airfoils - A Concise NURBS-based Scheme}

The development of the formulation presented here was driven by a 'wish-list' resulting from our desire to conduct airframe conceptual design in a Multidisciplinary Design Optimization (MDO) environment comprising Computer Aided design (CAD) engines, geometry-based conceptual design tools and a variety of numerical analysis packages (both stand-alone and CAD-integrated). The

9

Submitted to the American Institute of Aeronautics and Astronautics 
methodology described in this section was thus conceived as an answer to the following requirements, listed in decreasing order of importance:

1. Conciseness is paramount. Minimizing the problem dimensionality $k$ is the overriding objective, accepting losses in terms of flexibility. This means exploring the possibility of reducing the number of design variables to as low as one, placing the parametric airfoil we seek at the global extreme of the classification discussed above.

2. Non-uniform Rational B-Splines (NURBS) have become the standard formulation for geometry representation in most types of MDO-related software, from CAD, through meshing tools to analysis codes. While work-arounds exist for transferring non-B-Spline-based geometries into such tools, these processes are rarely error-free. Therefore we postulate that the representation must be NURBS-based.

3. In order to minimize the number of wasted automated analysis runs, the entire design space should be feasible. In other words, any combination of design variable values should produce 'airfoil-like' shapes.

4. The primary area of application is the design of transonic wings and the parametric airfoil should therefore have the ability to take up supercritical shapes.

5. Design is generally performed at increasing levels of detail. Therefore, multiple levels of flexibility should be built into the same parametric airfoil description.

We achieve the above by constructing a generic NURBS-based airfoil, which is able to imitate a range of existing supercritical sections. We then select two such imitations, which will serve as the bases of a fully global (as per the taxonomy presented in Section I), single variable parameterisation scheme and we outline a recipe for using this in a design context. We then consider two and three variable extensions.

\section{A Pair of Non-Uniform Rational B-Splines (NURBS)}

Rational B-splines are first mentioned in the $\mathrm{PhD}$ thesis of Versprille. ${ }^{16}$ Since then they have become the fundamental building block of most geometry standards and CAD tools and their use in design has been the subject of much research (see, for example, work by Schramm et al. ${ }^{17}$ and Samareh ${ }^{18}$ ) - hence our inclusion of them at the top of the airfoil parameterisation scheme criteria list. Here we shall use a pair of NURBS to define the upper and lower surfaces of an airfoil. Let the series of coordinate pairs $\left(x_{i}^{u}, y_{i}^{u}\right)$ and $\left(x_{i}^{l}, y_{i}^{l}\right)$, where $i=0, \ldots, 5$, define the control polygons of the two curves that form the airfoil and are of the form

$$
\mathbf{S}^{u}(t)=\frac{\sum_{i=0}^{5} N_{i, p}(t) w_{i}^{u}\left(x_{i}^{u}, y_{i}^{u}\right)}{\sum_{i=0}^{5} N_{i, p}(t) w_{i}^{u}}, \quad t \in[0,1]
$$

for the upper surface and

$$
\mathbf{S}^{l}(t)=\frac{\sum_{i=0}^{5} N_{i, p}(t) w_{i}^{l}\left(x_{i}^{l}, y_{i}^{l}\right)}{\sum_{i=0}^{5} N_{i, p}(t) w_{i}^{l}}, \quad t \in[0,1]
$$


for the lower surface. The two surfaces share the fixed, non-decreasing knot vector $T=\left\{t_{0}, \ldots, t_{9}\right\}$, which we use to define the same B-spline basis functions of degree $p$ (order $p+1$ ) that we used in Section III, which, for convenience, we repeat here:

$$
\begin{gathered}
N_{i, p}(t)=\frac{t-t_{i}}{t_{i+p}-t_{i}} N_{i, p-1}(t)+\frac{t_{i+p+1}-t}{t_{i+p+1}-t_{i+1}} N_{i+1, p-1}(t) . \\
N_{i, 0}(t)=\left\{\begin{array}{lcc}
1 & \text { if } & t_{i} \leq t<t_{i+1} \\
0 & \text { otherwise. }
\end{array}\right.
\end{gathered}
$$

For the definition of both the upper and the lower surfaces of the airfoil we set $p=2$ and

$$
T=\{0,0,0,0.25,0.5,0.75,1,1,1\}
$$

where the multiplicity of the extreme values indicates that the curve will actually pass through the first and the last control point (the leading edge point and the trailing edge point(s) in our case more on this shortly). The reader interested in why this is so and, indeed, in any other detail of the above, might find the standard text by Piegl and Tiller ${ }^{15}$ edifying.

Let the pair $\Omega(\mathbf{A})=\left[\mathbf{S}^{u}, \mathbf{S}^{l}\right]$ thus denote a NURBS aerofoil, where the matrix $\mathbf{A}$ provides an 'at-a-glance' view of of all the relevant parameters defining the airfoil (each column corresponds to a pair of control points on either side of the airfoil):

$$
\mathbf{A}=\left[\begin{array}{cccccc}
x_{0}^{u} & x_{1}^{u} & x_{2}^{u} & x_{3}^{u} & x_{4}^{u} & x_{5}^{u} \\
y_{0}^{u} & y_{1}^{u} & y_{2}^{u} & y_{3}^{u} & y_{4}^{u} & y_{5}^{u} \\
w_{0}^{u} & w_{1}^{u} & w_{2}^{u} & w_{3}^{u} & w_{4}^{u} & w_{5}^{u} \\
x_{0}^{l} & x_{1}^{l} & x_{2}^{l} & x_{3}^{l} & x_{4}^{l} & x_{5}^{l} \\
y_{0}^{l} & y_{1}^{l} & y_{2}^{l} & y_{3}^{l} & y_{4}^{l} & y_{5}^{l} \\
w_{0}^{l} & w_{1}^{l} & w_{2}^{l} & w_{3}^{l} & w_{4}^{l} & w_{5}^{l}
\end{array}\right]
$$

This gives us a total of 36 potential degrees of freedom, a number of which we choose to fix. First, the leading edge points of both surfaces will always be at $(0,0)$ - see Figure 5 . The chord length will also be fixed (at 1) and so will all the control point abscissas, with points 0 and 1 being fixed at $x=0$ and point 5 (on both sides) always having an abscissa of 1 . The remaining control points will be, in terms of their $x$-coordinates, uniformly distributed along the chord. These decisions halve the number of degrees of freedom contained in $\mathbf{A}$ and we shall, in what follows, refer to this constrained form of the variable definition matrix as

$$
\overline{\mathbf{A}}=\left[\begin{array}{cccccc}
0 & 0 & 0.25 & 0.5 & 0.75 & 1 \\
0 & y_{1}^{u} & y_{2}^{u} & y_{3}^{u} & y_{4}^{u} & y_{5}^{u} \\
1 & w_{1}^{u} & w_{2}^{u} & w_{3}^{u} & w_{4}^{u} & 1 \\
0 & 0 & 0.25 & 0.5 & 0.75 & 1 \\
0 & y_{1}^{l} & y_{2}^{l} & y_{3}^{l} & y_{4}^{l} & y_{5}^{l} \\
1 & w_{1}^{l} & w_{2}^{l} & w_{3}^{l} & w_{4}^{l} & 1
\end{array}\right]
$$

These 18 remaining variables included in $\overline{\mathbf{A}}$ are shown in Figure 5.

11 


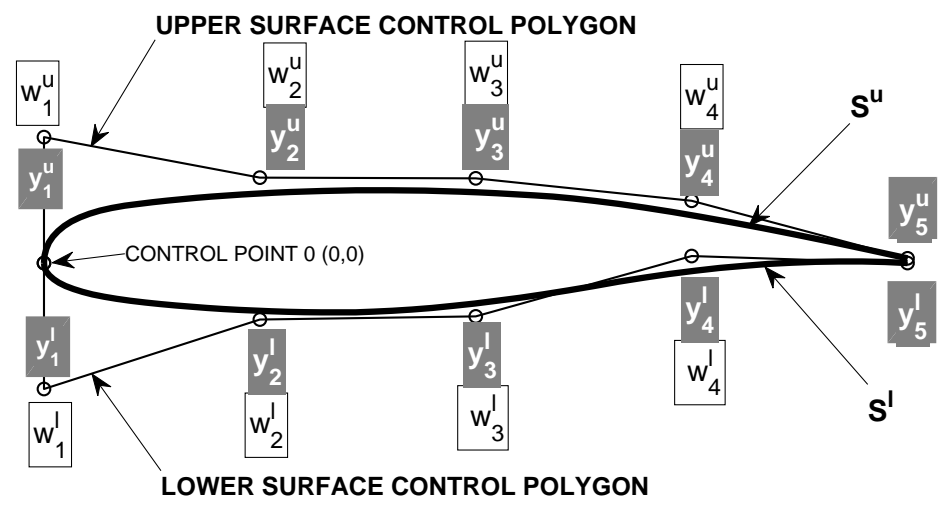

Fig. 5 The control point weights (framed) and ordinates (on gray background) through which we determine the shape of the NURBS-based airfoil.

\section{Constructing Imitations}

The parametric geometrical construction comprising the two NURBS curves, whose parameters are defined by $\overline{\mathbf{A}}$, will form the starting point of our concise, conceptual-level parametric airfoil. More specifically, we shall deploy its ability to approximate existing airfoils in the service of capturing knowledge about well-known supercritical airfoil shapes. Of course, what we have so far is, in itself, a parametric airfoil and, while it has too many parameters for conceptual design purposes, it is flexible enough to be capable of 'morphing' into rather close imitations of a range of standard, well-proven, existing airfoils - it is this feature that we exploit in what follows.

For a selected range of 'classic' airfoils, we seek sets of parameters for each, that, when plugged into the matrix $\overline{\mathbf{A}}$ that defines the NURBS airfoil, this will approximate them to within as small a margin as possible. We use the overlapping areas to compute this margin. To be precise, we define the difference between a classic airfoil and its NURBS approximation as the sum of the areas between the two airfoils aligned such that their chords overlap, expressed as a percentage of the total area of the 'classic' airfoil. Note that this is not the difference between the areas of the target and the approximation, as that could be near zero and the approximation could still 'snake' undetected around the target airfoil, as long as the overshoots roughly cancel out the undershoots. Figure 6 illustrates a case of (exagerated) snaking and highlights the area we are actually using as a difference metric.

We shall denote this difference by $d[\omega, \Omega(\overline{\mathbf{A}})]$, referring to the difference between some airfoil $\omega$ and a NURBS-based foil $\Omega(\overline{\mathbf{A}})$. With a difference metric in place, we can now formalize our notion of the best NURBS-based approximation of an existing airfoil.

Definition $1 \Omega_{\omega}=\Omega(\overline{\mathbf{A}})$ is the NURBS imitation of the airfoil $\omega$ iff

$$
\forall \overline{\mathbf{A}}^{*} \neq \overline{\mathbf{A}}, \quad \mathrm{d}[\Omega(\overline{\mathbf{A}}), \omega] \leq \mathrm{d}\left[\Omega\left(\overline{\mathbf{A}}^{*}\right), \omega\right]
$$




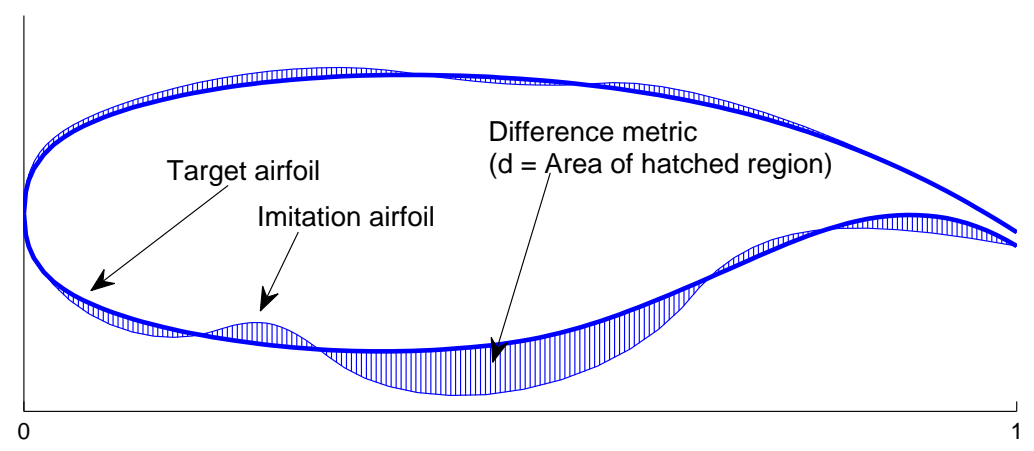

Fig. 6 The hatched area is representative of the difference between a target airfoil and its imitation. $d$ is this area, expressed as a percentage of the area of the target airfoil (depicted here by the heavy black line contour).

It is worth pointing out here that the minimization problem of Definition 1 and thus the finding of the NURBS imitation is usually not a trivial exercise, owing to the multi-modal nature of the difference function $d$. The dimensionality of this landscape is also relatively high, as even the restricted $\overline{\mathbf{A}}$ has 18 variables. Thus, once a minimum has been found, however diligent we may have been in optimizing $d$, it is impossible to be absolutely certain that we have indeed located the global minimum. As a reminder of this, when using the notation introduced above, we shall precede the name of the classic airfoil with the 'approximately equal' symbol. For example, we shall use $\Omega_{\sim \mathrm{SC}(2)-0714}$ to refer to the best NURBS imitation of the NASA supercritical airfoil SC(2)-0714 we could find through a numerical optimization procedure.

As per criterion 4 of our wish-list, we shall focus on a range of supercritical airfoils. To begin with, let us consider eight well-known examples, which, we feel, are representative of this class. What they all share is the goal that drove their conception, which is to maximize the drag-rise Mach number and thus maximize the cruising speed of an aircraft whose wings are based on these sections. First, we have considered five of the NASA Phase 2 supercritical airfoils, the $\mathrm{SC}(2)$ family. ${ }^{10}$ Three of these are the 'thin', 6\% thickness to chord ratio foils, $\mathrm{SC}(2)-0406, \mathrm{SC}(2)-0606$ and $\mathrm{SC}(2)-0706$, designed for lift coefficients of $0.4,0.6$ and 0.7 respectively. The fourth is the much-studied $\mathrm{SC}(2)-0714,{ }^{19}$ while the final member of this group is the $18 \%$ thick $\mathrm{SC}(2)-0518$. Another high thickness to chord ratio airfoil we have included is the Dutch National Aerospace Laboratory NLR7301. It is slightly unusual amongst supercritical airfoils not only by virtue of its $16.3 \%$ maximum thickness, but also because of its rather large nose radius. A 1975 paper by Boerstoel and Huizing ${ }^{20}$ discusses in detail the hodograph calculations used in its design; the reader interested in detailed test results on it can find them, for example, in an extensive AGARD report. ${ }^{21}$ The same report contains test data regarding the RAE 2822, also included in our set. Finally, we use the RAE5215, featuring a thickness to chord ratio of $9.7 \%$. It is the result of alterations made in the early 1970 s to two earlier RAE sections (5213 and 5214), the chief aim of this development work being the elimination of rear separation at low Reynolds numbers. ${ }^{22}$ 

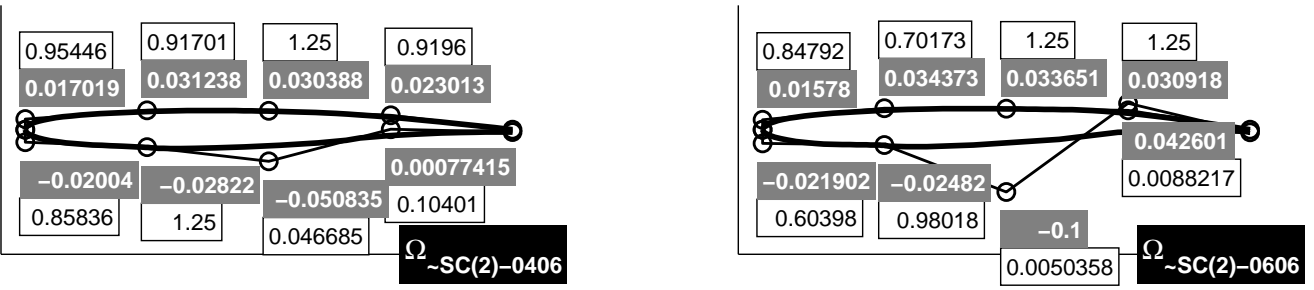

$\mathrm{d}=5.8156 \%$
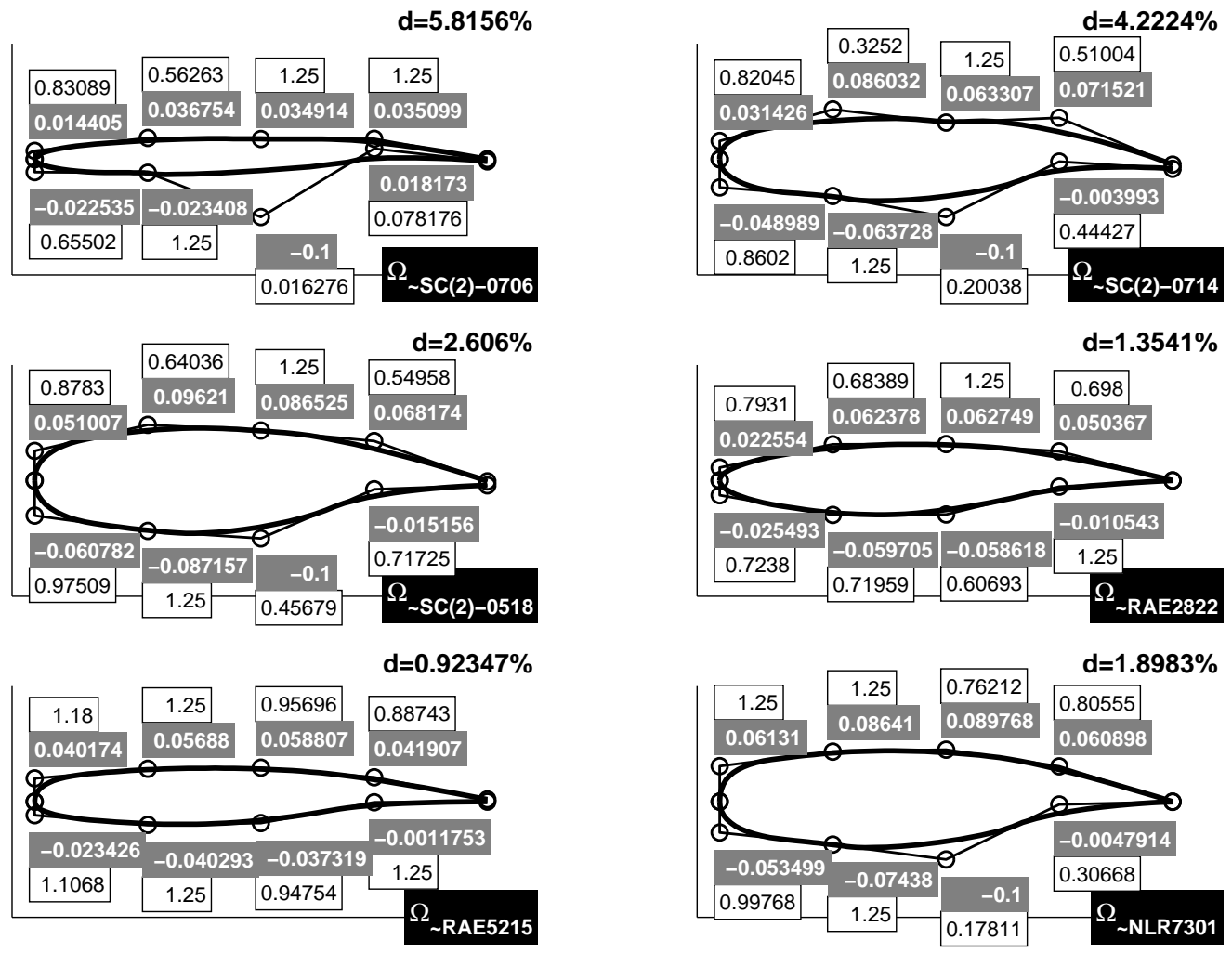

Fig. 7 The NURBS imitations of eight classic airfoils and the differences $d$ between them and their 'real' counterparts. The axes are not to the same scale. 
We mentioned earlier the challenges of optimizing the difference metric $d$. There is a broad range of optimizers that could be used to find the $\Omega_{\sim \omega} \mathrm{s}$. We have experimented on the present set of functions with two potential candidates. First, a Nelder and Mead simplex pattern search was tested, see Ref. 23 for notes on the implementation used. Secondly, we considered a BFGS search (see, e.g., Ref. 24) - this local, quasi-Newton optimization procedure estimating here the landscape gradients through finite differencing (these are needed, in turn, for the approximation of the Hessian $\mathrm{H}(d)$ ). Of these two, the BFGS required fewer evaluations of $d$ in these tests.

To reduce the size of the search space we have set the values of the top and bottom ordinates of the trailing edge points in the NURBS approximation ( $y_{5}^{u}$ and $y_{5}^{l}$ respectively) to those of the target, classic airfoils we were attempting to imitate, thus cutting the dimensionality down to 16 . To further simplify the search problem we have implemented a two stage approach. First, all weights were fixed at 1 and $d$ was optimized subject to the control point ordinates. The second, fine-tuning stage then allowed the ordinates, as well as the weights to vary.

Figure 7, a graphical depiction of the results of the search for the imitations of our eight chosen airfoils, shows these optimized sets of 16 numbers, as well as the corresponding difference values.

What these imitations show is that the definition of the two NURBS curves that make up the airfoil is flexible enough to provide good approximations of a fairly representative set of supercritical airfoils. We shall use these imitations (or, in the spirit of our introductory discussion, supercritical airfoil-likeness knowledge capture devices) as the pillars of our parsimonious, conceptual level parameterization scheme, which we discuss next.

\section{Linear Combinations and a Single Variable Parameterization Scheme}

In the introduction we have underlined the importance of being parsimonious with the geometric parameterisation of a conceptual airframe model and we made this our top priority. In that spirit, we shall now consider a NURBS-based formulation requiring a single 'slider control' variable to sweep the span between two of the imitations we have prepared earlier. As we noted earlier, these imitations represent our knowledge (incomplete as it may be) of what makes a pair of NURBS a supercritical airfoil.

The essence of deploying this knowledge is simple: we re-parameterise the matrix $\overline{\mathbf{A}}$ as the linear combination of two (imitations of) classics, with a weighting parameter $\alpha$ controlling the relative contributions of these two base airfoils. In order to formalise this we define two new operators.

Definition 2 We define the binary operator $\star$ on the space of matrices of the form $\overline{\mathbf{A}}$, such that $\forall \alpha \in \mathbb{R}: \alpha \star \overline{\mathbf{A}}=\Gamma_{\star}(\alpha) \bullet \overline{\mathbf{A}}$, where $\bullet$ is the Haddamard (entry-wise) matrix product and

$$
\Gamma_{\star}(\alpha)=\left[\begin{array}{cccccc}
1 & 1 & 1 & 1 & 1 & 1 \\
1 & \alpha & \alpha & \alpha & \alpha & \alpha \\
1 & \alpha & \alpha & \alpha & \alpha & 1 \\
1 & 1 & 1 & 1 & 1 & 1 \\
1 & \alpha & \alpha & \alpha & \alpha & \alpha \\
1 & \alpha & \alpha & \alpha & \alpha & 1
\end{array}\right]
$$

15 
Definition 3 We define the binary operator $\oplus$ on the space of matrices of the form $\overline{\mathbf{A}}$, such that $\forall \overline{\mathbf{A}_{1}}, \overline{\mathbf{A}_{\mathbf{2}}}: \overline{\mathbf{A}_{\mathbf{1}}} \oplus \overline{\mathbf{A}_{\mathbf{2}}}=\mathbf{A}_{\mathbf{1}}+\Gamma_{\oplus} \bullet \overline{\mathbf{A}_{\mathbf{2}}}$, where $\bullet$ is the Haddamard (entry-wise) matrix product and

$$
\Gamma_{\oplus}=\left[\begin{array}{llllll}
0 & 0 & 0 & 0 & 0 & 0 \\
0 & 1 & 1 & 1 & 1 & 1 \\
0 & 1 & 1 & 1 & 1 & 0 \\
0 & 0 & 0 & 0 & 0 & 0 \\
0 & 1 & 1 & 1 & 1 & 1 \\
0 & 1 & 1 & 1 & 1 & 0
\end{array}\right]
$$

The matrices $\Gamma_{\star}(\alpha)$ and $\Gamma_{\oplus}$ are, essentially, stencils that control the elements of the generic parameterization that will be altered during the linear combination operation, which we define next.

Definition 4 Consider the NURBS imitations of some airfoils $\omega 1$ and $\omega 2$, denoted by $\Omega_{\omega 1}=$ $\Omega\left(\overline{\mathbf{A}}_{\omega 1}\right)$ and $\Omega_{\omega 2}=\Omega\left(\overline{\mathbf{A}}_{\omega 2}\right)$ respectively (i.e., $\overline{\mathbf{A}}_{\omega 1}$ and $\overline{\mathbf{A}}_{\omega 2}$ contain the variables that define the two imitations). We shall refer to the single-variable, parametric airfoil

$$
\Omega_{\omega 1}^{\omega 2}(\alpha)=\Omega\left[\alpha \star \overline{\mathbf{A}}_{\omega 1} \oplus(1-\alpha) \star \overline{\mathbf{A}}_{\omega 2}\right]
$$

where $\alpha \in[0,1]$, as a linear combination NURBS airfoil over the bases $\omega 1$ and $\omega 2$.

The question arising now is, which bases $\omega 1$ and $\omega 2$ would ensure the best coverage of the design space as $\alpha$ sweeps its range? We have the eight imitations of Section IV to choose from - the question is, how do we measure the suitability of a given pair? As per criterion 4, for a given $k$ we aim to maximize the ability of the parametric airfoil to approximate existing supercritical sections. We can estimate this ability, using the overlapping areas once again as the difference metric in the same way as we did with the imitations, by attempting to approximate a set of test sections, representative of the supercritical family. In addition to the eight potential base sections, we have also included in this test set four more NASA phase 2 supercriticals for a better representation of the mid-range of thickness to chord ratios: $\mathrm{SC}(2)-0410, \mathrm{SC}(2)-0710, \mathrm{SC}(2)-0412$ and $\mathrm{SC}(2)-0712$. We have evaluated the comparative abilities of the $C_{8}^{2}=28$ possible basis pairs to represent the 12 airfoils in this set. We have found the 'partnership' of NLR7301 and SC(2)-0606 to work best. More formally, the evaluation of

$$
\min \left\{\sum_{i=1}^{12} \min _{\alpha} d\left[\omega_{i}, \Omega_{\omega_{\text {base } 1}}^{\omega_{\text {base }}}(\alpha)\right] \mid\left(\omega_{\text {base } 1}, \omega_{\text {base } 2}\right) \subset B\right\}
$$

resulted in $\left(\omega_{\text {base } 1}, \omega_{\text {base } 2}\right)=(\mathrm{NLR} 7301, \mathrm{SC}(2)-0606)$, where $\omega_{1}=\mathrm{SC}(2)-0406, \omega_{2}=\mathrm{SC}(2)-0606, \ldots \omega_{12}=$ NLR7301 are the test airfoils (see the legend of Figure 8 for the full list) and

$$
B=\{\mathrm{SC}(2)-0406, \mathrm{SC}(2)-0606, \ldots \mathrm{NLR} 7301\}
$$

is the list of the eight potential bases (as shown in Figure 7).

This is not a particularly surprising outcome, as one would intuitively expect the process to yield one of the thinnest and one of the thickest airfoils as the best pillars, though it would have been 


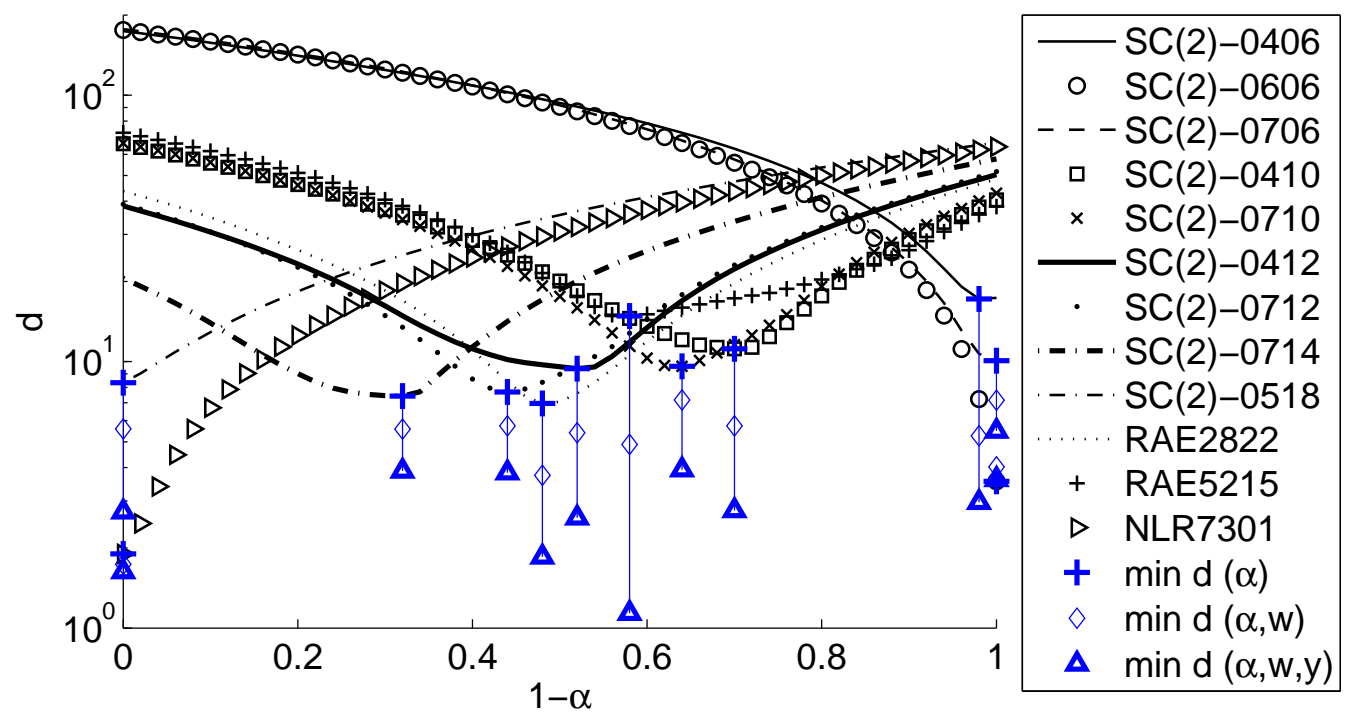

Fig. 8 Plots of $d\left[\omega_{i}, \Omega_{\sim N L R 7301}^{\sim S C(2)-0606}(\alpha)\right]$ for $\omega_{1}=\mathbf{S C}(2)-0406, \omega_{2}=\mathbf{S C}(2)-0606, \ldots \omega_{12}=\mathbf{N L R 7 3 0 1}$. The ' + ' symbols indicate the minimum of each curve, i.e., the ordinates of these indicate the minimum difference to within which the linear combination of NLR7301 and SC(2)-0606 can approximate the 12 foils in our test set. The diamond symbols indicate the minimum difference achievable through a local search started from that airfoil, which allows the control point weights to vary, while the ordinates of the triangles indicate the minimum $d$ achievable by also allowing the control point ordinates to change during a single start local search.

harder to predict exactly which of the two thick and which of the three thin foils would provide the best 'supercritical airfoil-ness' knowledge representation. Approximating the 12 members of the test set with linear combinations of the chosen pair gave an average minimum difference of

$$
\frac{1}{12} \sum_{i=1}^{12} \min _{\alpha} d\left[\omega_{i}, \Omega_{\sim \mathrm{NLR} 7301}^{\sim \mathrm{SC}(2)-0606}(\alpha)\right]=9.0601 \% .
$$

This experiment is captured graphically in Figure 8. The difference $(d)$ curves corresponding to each test airfoil can be seen dipping, for an optimum value of the linear combination factor $\alpha$, to a minimum - this is the point where the parametric airfoil $\Omega_{\sim N L R 7301}^{\sim S C(2)-0606}(\alpha)$ resembles the target (test) airfoil most closely. For example,

$$
\Omega_{\sim \mathrm{NLR} 7301}^{\sim \mathrm{SC}(2)-0606}(0.675)=\Omega\left[0.675 \star \overline{\mathbf{A}}_{\sim \mathrm{NLR} 7301} \oplus 0.325 \star \overline{\mathbf{A}}_{\sim \mathrm{SC}(2)-0606}\right]
$$

is the best approximation of $\mathrm{SC}(2)-0714(d=7.1993)$ that can be found using this scheme (we have run a direct search over the $\alpha$ domain with a resolution of $10^{-2}$ here - the curves show that there is little to be gained by employing a higher resolution search). 
It is worth emphasizing once more that, in the spirit of our list of requirements postulated in the introduction, we did not aim (and, indeed, cannot really hope) for extremely accurate approximations here. Instead, we are exploring what is the best we can do with a single design variable available for controlling a parametric supercritical airfoil, while ensuring the satisfaction of the four other items of our wish list from the beginning of Section V. On that note, let us now review the significance of the above discussion from the conceptual design standpoint, which is at the centre of this paper.

\section{Designing with Linear Combination NURBS Airfoils}

Let $v_{1}, v_{2}, \ldots v_{k}$ be a set of design variables that define an airframe geometry. As we are focusing on airfoil definition here, let $v_{1}$ be the single parameter we are using to define, say, the wing root section. We do this as described above by building a control polygon and a pair of NURBS curves (as depicted in Figure 5), with the numbers needed to do this contained in the definition matrix, calculated in terms of the design variable $v_{1}$ as

$$
\overline{\mathbf{A}}=v_{1} \star \overline{\mathbf{A}}_{\sim \mathrm{NLR} 7301} \oplus\left(1-v_{1}\right) \star \overline{\mathbf{A}}_{\sim \mathrm{SC}(2)-0606}
$$

For convenience we split the airframe description into the airfoil thus defined, $\Omega_{\sim \mathrm{NLR}(2)-0606}^{\sim \mathrm{SC}}\left(v_{1}\right)$, and $G\left(v_{2}, v_{3}, \ldots v_{k}\right)$, the latter denoting the rest of the geometry.

Consider now the functional $\mathcal{F}\left[\Omega_{\sim \mathrm{NLR7301}}^{\sim \mathrm{SC}(2)-0606}\left(v_{1}\right), G\left(v_{2}, v_{3}, \ldots v_{k}\right)\right]$ mapping some figure of merit to the airframe geometry. Typically, this is an objective (computed through a numerical simulation), which we seek to maximize or minimize. An example is total airframe drag at the angle of attack where the total lift equals the all-up weight. Thus, the conceptual design search can be boiled down to the minimization problem

$$
\min _{v_{1} \in[0,1], v_{2}, \ldots v_{k}} \mathcal{F}\left[\Omega_{\sim \mathrm{NLR} 7301}^{\sim \mathrm{SC}(2)-0606}\left(v_{1}\right), G\left(v_{2}, v_{3}, \ldots v_{k}\right)\right],
$$

to whose dimensionality the airfoil only contributes one.

The linear combination NURBS airfoil essentially represents a continuum of supercritical airfoils between the two bases, whose imitations it reproduces exactly at the extreme values of $v_{1}$ (NLR7301 at $v_{1}=0$ and $\mathrm{SC}(2)-0606$ at $\left.v_{1}=1\right)$. Therefore, the optimization problem (29) can be solved with any continuous variable optimization algorithm. As an aside, the same geometric construction can be used for a search over a given catalog of imitations, rather than over the continuum bridging the gap between two imitations. For example, if we take the set $B$ of our eight imitated classic airfoils (those shown in Figure 7) as the catalog, the problem can be re-cast as:

$$
\min _{v_{1} \in\{1,2 \ldots, 8\}, v_{2}, \ldots v_{k}} \mathcal{F}\left[\Omega_{\sim B\left(v_{1}\right)}, G\left(v_{2}, v_{3}, \ldots v_{k}\right)\right]
$$

which means that the search will now be discrete-valued along the dimension $v_{1}$.

Returning now briefly to Figure 8, earlier we left unexplained part of the experiment depicted therein and this is the appropriate moment to return to it. For every test airfoil, once we have established the weighting that brings the parametric linear combination NURBS airfoil nearest to it, we froze the weighting at that value and further minimized the difference $d$ using a Nelder and Mead 
simplex pattern search, this time subject to the control point weights. The optima of these eightdimensional local search processes can be read off the chart as the ordinates of the diamond symbols (note that these are not the globally best approximations that can be obtained for the various test airfoils, merely the minima of the local basins of attraction). Next, we ran the Nelder and Mead search once more, this time allowing both the weights and the control point ordinates to vary, giving the minima whose difference values are the ordinates of the triangle symbols.

This logic could also be applied to the optimization of $\mathcal{F}$ at the later stages of the development process, perhaps as a preliminary design operation, where we are willing to trade increased dimensionality for potentially better objective functional value. Defining the stencil matrix

$$
\Gamma_{w}=\left[\begin{array}{llllll}
0 & 0 & 0 & 0 & 0 & 0 \\
0 & 0 & 0 & 0 & 0 & 0 \\
0 & 1 & 1 & 1 & 1 & 0 \\
0 & 0 & 0 & 0 & 0 & 0 \\
0 & 0 & 0 & 0 & 0 & 0 \\
0 & 1 & 1 & 1 & 1 & 0
\end{array}\right]
$$

we can formalize this operation as

$$
\min _{\Gamma_{w} \bullet \mathbf{A}, v_{2}, \ldots v_{k}} \mathcal{F}\left[\Omega_{\sim \operatorname{NLR} 7301}^{\sim \mathrm{SC}(2)-0606}\left(v_{1}^{\mathrm{opt}}\right), G\left(v_{2}, v_{3}, \ldots v_{k}\right)\right],
$$

where we have frozen $v_{1}$ at $v_{1}^{\text {opt }}$, the optimum value found during the conceptual design process and we are now allowing the control point weights to be subjected to the optimization process. Finally, mirroring the experiments of Figure 8 in a real, design optimization context, we can now turn on all of the degrees of freedom built into our NURBS airfoil definition by allowing every entry in $\overline{\mathbf{A}}$ to vary in an optimization process that can be summarized as:

$$
\underset{\overline{\mathbf{A}}, v_{2}, \ldots v_{k}}{\min } \mathcal{F}\left[\Omega_{\sim \mathrm{NLR} 7301}^{\sim \mathrm{SC}(2)-0606}\left(v_{1}^{\mathrm{opt}}\right), G\left(v_{2}, v_{3}, \ldots v_{k}\right)\right] .
$$

At this level the formulation proposed here becomes very similar to those described by Lépine et al. ${ }^{6}$ and Painchaud-Ouellet et al. ${ }^{7}$ In terms of meeting our initial criteria listed at the beginning of Section $\mathrm{V}$, these last two preliminary design type steps now have increasing dimensionalities, which are hard to justify against criterion one. Also, they depart from the third criterion of our wish list, as the parameterisation can now yield 'unphysical' shapes too (although these are fairly unlikely to occur, as an aerodynamics-based $\mathcal{F}$ will probably still favor smooth, 'reasonable' shapes). We have, however, used the same geometrical entities for these different levels of optimization (criterion five), by merely varying the method whereby the control point weights and ordinates are calculated. In other words, the same model can be used throughout the entire design process, offering potential benefits in terms of development cost reduction.

Let us now return to the pure linear combination approach by considering the possibility of incorporating additional knowledge into the parametric foil, simply by using additional learning cases. 


\section{Learning from More Examples}

Let us consider the potential flexibility enhancements that additional base airfoils (as further learning examples) would buy us. To this end, it is fairly straightforward to conceive an extension of Definition 4, as follows.

Definition 5 Consider the NURBS imitations of four airfoils $\omega 1, \omega 2, \omega 3$ and $\omega 4$, denoted by $\Omega_{\omega 1}=\Omega\left(\overline{\mathbf{A}}_{\omega 1}\right), \Omega_{\omega 2}=\Omega\left(\overline{\mathbf{A}}_{\omega 2}\right), \Omega_{\omega 3}=\Omega\left(\overline{\mathbf{A}}_{\omega 3}\right)$ and $\Omega_{\omega 4}=\Omega\left(\overline{\mathbf{A}}_{\omega 4}\right)$ respectively (i.e., $\overline{\mathbf{A}}_{\omega 1}, \overline{\mathbf{A}}_{\omega 2}, \overline{\mathbf{A}}_{\omega 3}$ and $\overline{\mathbf{A}}_{\omega 4}$ contain the variables that define the four imitations). We shall refer to the parametric airfoil

$$
{ }_{\omega 1} \Omega_{\omega 2}^{\omega 3}\left(\alpha_{1}, \alpha_{2}, \alpha_{3}\right)=\Omega\left[\alpha_{1} \star \overline{\mathbf{A}}_{\omega 1} \oplus \alpha_{2} \star \overline{\mathbf{A}}_{\omega 2} \oplus \alpha_{3} \star \overline{\mathbf{A}}_{\omega 3}\right]
$$

where $\alpha_{1}, \alpha_{2}, \alpha_{3} \in \mathbb{R}^{+}$and $\alpha_{1}+\alpha_{2}+\alpha_{3}=1$, as a linear combination NURBS airfoil over the bases $\omega 1, \omega 2$ and $\omega 3$ and to the parametric airfoil

$$
{ }_{\omega 1}^{\omega 4} \Omega_{\omega 2}^{\omega 3}\left(\alpha_{1}, \alpha_{2}, \alpha_{3}, \alpha_{4}\right)=\Omega\left[\alpha_{1} \star \overline{\mathbf{A}}_{\omega 1} \oplus \alpha_{2} \star \overline{\mathbf{A}}_{\omega 2} \oplus \alpha_{3} \star \overline{\mathbf{A}}_{\omega 3} \oplus \alpha_{4} \star \overline{\mathbf{A}}_{\omega 4}\right]
$$

where $\alpha_{1}, \alpha_{2}, \alpha_{3}, \alpha_{4} \in \mathbb{R}^{+}$and $\alpha_{1}+\alpha_{2}+\alpha_{3}+\alpha_{4}=1$, as a linear combination NURBS airfoil over the bases $\omega 1, \omega 2, \omega 3$ and $\omega 4$.

The problem of approximating a given target airfoil with a linear combination NURBS airfoil over three bases is virtually the same as with two bases. The only exception is that the optimization problem is now subject to three $\alpha$ 's instead of one. The overall dimensionality is reduced from three to two by the additional equality constraint, though in most cases, from a computational implementation standpoint, the problem is more readily viewed as three dimensional and constrained, rather than projected onto the two-dimensional plane $\alpha_{1}+\alpha_{2}+\alpha_{3}=1\left(\alpha_{1}, \alpha_{2}, \alpha_{3} \in \mathbb{R}^{+}\right)$and unconstrained (see the homomorphous mapping approach of Monson and $\mathrm{Seppi}^{25}$ for taking this latter route, if preferred).

Once again, we need to select the bases that, with their $\alpha$ 's optimized, best approximate the other members of our representative set of 12 test airfoils (this being a simple test of the satisfaction of criterion 4). Formally, we are seeking the solution of the two level minimization problem

$$
\min \left\{\sum_{i=1}^{12} \min _{\alpha_{1}, \alpha_{2}, \alpha_{3}} d\left[\omega_{i}, \omega_{\text {base } 1} \Omega_{\omega_{\text {base } 2}}^{\omega_{\text {base }}}\left(\alpha_{1}, \alpha_{2}, \alpha_{3}\right)\right] \mid\left(\omega_{\text {base } 1}, \omega_{\text {base } 2}, \omega_{\text {base } 3}\right) \subset B\right\},
$$

where all $C_{8}^{3}=56$ subsets of three of the set of eight potential base airfoils are tested across the set of 12 test airfoils. A direct search solve of (34) yielded

$$
\left(\omega_{\mathrm{base} 1}, \omega_{\mathrm{base} 2}, \omega_{\mathrm{base} 3}\right)=(\mathrm{RAE} 5215, \mathrm{SC}(2)-0518, \mathrm{SC}(2)-0606)
$$

with the difference metric averaging $7.0315 \%$ over the 12 target sections, which is around three quarters of the estimated average approximation error of the two basis airfoil scheme (9.0601\%). To single out one particular example, the difference metric between $\mathrm{SC}(2)-0714$ and its best approximation was 


\begin{tabular}{|c|c|c|c|c|}
\hline$\omega$ & $\alpha_{1}$ & $\alpha_{2}$ & $\alpha_{3}$ & $d\left[\omega, \sim R A E 5215 \Omega_{\sim S C(2)-0518}^{\sim S C(2)-0606}\left(\alpha_{1}, \alpha_{2}, \alpha_{3}\right)\right]$ \\
\hline \hline $\mathrm{SC}(2)-0406$ & 0.0042 & 0 & 0.9958 & $12.3886 \%$ \\
$\mathrm{SC}(2)-0606$ & 0 & 0 & 1 & $4.1986 \%$ \\
$\mathrm{SC}(2)-0706$ & 0 & 0 & 1 & $11.3089 \%$ \\
$\mathrm{SC}(2)-0410$ & 0 & 0.2669 & 0.7331 & $9.1881 \%$ \\
$\mathrm{SC}(2)-0710$ & 0.7072 & 0.0930 & 0.1997 & $10.7707 \%$ \\
$\mathrm{SC}(2)-0412$ & 0 & 0.4496 & 0.5504 & $6.7767 \%$ \\
$\mathrm{SC}(2)-0712$ & 0.6030 & 0.3121 & 0.0849 & $8.0071 \%$ \\
$\mathrm{SC}(2)-0714$ & 0.1237 & 0.5962 & 0.2801 & $4.6457 \%$ \\
$\mathrm{SC}(2)-0518$ & 0 & 1 & 0 & $2.6060 \%$ \\
$\mathrm{RAE} 2822$ & 0.5172 & 0.3002 & 0.1826 & $7.5347 \%$ \\
$\mathrm{RAE} 5215$ & 1 & 0 & 0 & $0.9235 \%$ \\
$\mathrm{NLR} 7301$ & 0.2155 & 0.7845 & 0 & $6.0293 \%$ \\
\hline
\end{tabular}

Table 1 The differences between the test set of twelve airfoils and the closest linear combination NURBS airfoils over the bases RAE5215, SC(2)-0518 and SC(2)-0606, obtained by minimizing $d$ subject to $\alpha_{1}, \alpha_{2}$ and $\alpha_{3}$ (where $\alpha_{1}+\alpha_{2}+\alpha_{3}=1$ ).

found to be

$$
\begin{aligned}
& d\left[\mathrm{SC}(2)-0714, \quad \sim \operatorname{RAE} 5215 \Omega_{\sim \mathrm{SC}(2)-0518}^{\sim \mathrm{SC}(2)-066}(0.1237,0.5962,0.2801)\right]= \\
& =d\left[\mathrm{SC}(2)-0714, \quad \Omega\left(0.1237 \star \overline{\mathbf{A}}_{\sim \operatorname{RAE} 5215} \oplus 0.5962 \star \overline{\mathbf{A}}_{\sim \mathrm{SC}(2)-0518} \oplus 0.2801 \star \overline{\mathbf{A}}_{\sim \mathrm{SC}(2)-0606}\right)\right]= \\
& =4.6457 \% \text {, }
\end{aligned}
$$

approximately two thirds of the difference metric value of the two basis approximation of SC(2)-0714 (see equation (27)). Table 1 contains the full results of this experiment. Repeating now the exercise for four bases, we seek the solution of the two level minimization problem

$$
\min \left\{\sum_{i=1}^{12} \min _{\alpha_{1}, \alpha_{2}, \alpha_{3}, \alpha_{4}} d\left[\omega_{i}, \omega_{\omega_{\text {base }}}^{\omega_{\text {base }}} \Omega_{\omega_{\text {base } 2}}^{\omega_{\text {base3 }}}\left(\alpha_{1}, \alpha_{2}, \alpha_{3}, \alpha_{4}\right)\right] \mid\left(\omega_{\text {base1 } 1}, \omega_{\text {base2 } 2}, \omega_{\text {base3 } 3}, \omega_{\text {base4 }}\right) \subset B\right\},
$$

where now the $C_{8}^{4}=70$ subsets of four of the set of eight potential base airfoils are evaluated as potential bases over the set of 12 test airfoils. From a direct search solve of (34) results

$$
\left.\left(\omega_{\text {base } 1}, \omega_{\text {base } 2}, \omega_{\text {base3 }}, \omega_{\text {base } 4}\right)=(\operatorname{RAE} 5215, \mathrm{SC}(2)-0518, \operatorname{SC}(2)-0706), \operatorname{SC}(2)-0406\right),
$$

with the averaged difference metric now dropping to $4.9874 \%$ - comparing this to the best we could achieve with only two basis airfoils (just under twice this difference metric value, 9.0601\%) confirms the increase in the amount of additional geometrical features incorporated into the 'knowledge base' of the parametric airfoil.

\section{Conclusions and Possible Extensions}

We have presented four parametric airfoils, which, in our opinion, are suitable for conceptual design purposes, on account of their low dimensionality. First we have looked at three simple schemes defined 


\begin{tabular}{|c|c|c|c|c|c|}
\hline$\omega$ & $\alpha_{1}$ & $\alpha_{2}$ & $\alpha_{3}$ & $\alpha_{4}$ & $d\left[\omega, \underset{\sim R A E(2)-0406}{\sim} \Omega_{\sim S C(2)-0518}^{\sim S C(2)-0706}\left(\alpha_{1}, \alpha_{2}, \alpha_{3}, \alpha_{4}\right)\right]$ \\
\hline \hline $\mathrm{SC}(2)-0406$ & 0 & 0 & 0 & 1 & $2.1088 \%$ \\
$\mathrm{SC}(2)-0606$ & 0 & 0 & 0.8886 & 0.1114 & $5.8129 \%$ \\
$\mathrm{SC}(2)-0706$ & 0 & 0 & 1 & 0 & $5.8156 \%$ \\
$\mathrm{SC}(2)-0410$ & 0 & 0.2968 & 0.1673 & 0.5359 & $4.0453 \%$ \\
$\mathrm{SC}(2)-0710$ & 0.6250 & 0.1250 & 0.1250 & 0.1250 & $10.5663 \%$ \\
$\mathrm{SC}(2)-0412$ & 0 & 0.4691 & 0.1431 & 0.3877 & $3.5596 \%$ \\
$\mathrm{SC}(2)-0712$ & 0.5464 & 0.3378 & 0.0032 & 0.1124 & $7.9753 \%$ \\
$\mathrm{SC}(2)-0714$ & 0.0245 & 0.6323 & 0.1302 & 0.2130 & $3.8678 \%$ \\
$\mathrm{SC}(2)-0518$ & 0 & 1 & 0 & 0 & $2.6060 \%$ \\
$\mathrm{RAE} 2822$ & 0.4240 & 0.3417 & 0.0964 & 0.1380 & $6.8773 \%$ \\
$\mathrm{RAE} 5215$ & 1 & 0 & 0 & 0 & $0.9235 \%$ \\
$\mathrm{NLR7301}$ & 0.2155 & 0.7845 & 0 & 0 & $6.0055 \%$ \\
\hline
\end{tabular}

Table 2 The differences between the test set of twelve airfoils and the closest linear combination NURBS airfoils over the bases RAE5215, SC(2)-0518, SC(2)-0706 and SC(2)-0406 obtained by minimizing $d$ subject to $\alpha_{1}, \alpha_{2}, \alpha_{3}$ and $\alpha_{4}$ (where $\alpha_{1}+\alpha_{2}+\alpha_{3}+\alpha_{4}=1$ ).

by six variables each, which, while being easy to implement, are also sufficiently flexible to cover a range of 'classic' airfoils.

We then put forward a NURBS-based parametric airfoil specifically conceived for situations when minimizing the number of design variables is the overwhelming driver and the target application is transonic wings. We began by looking at the absolute limit of conciseness: a single variable formulation. This parametric airfoil is a linear combination of two, carefully selected, well-established supercritical shapes - the sole variable controls the relative contributions of these two basis sections to the parameterised shape. Clearly, the flexibility of this airfoil description is limited, but, as it can produce a range of airfoils of different thickness to chord ratios, it allows, for example, one of the fundamental types of MDO study at the conceptual design level: the investigation of the optimum trade-off between spar thickness, fuel tank capacity (assuming that the fuel is carried inside the wing) and drag.

More refined studies across a broader design space are possible using additional bases - we have shown that adding one or two additional basis sections into the linear combination increases the flexibility (and thus design space coverage) of the parametric airfoil. Further, we have demonstrated, that the same geometrical setup used in the linear combination scheme can be 'recycled' for preliminary design studies, where, if we sacrifice parsimony, extra flexibility is available for local optimization purposes, without the need for re-building the model.

The general thrust of the approach described here can be adapted for other types of geometry, including NURBS surfaces. For instance, it would be possible to fit a NURBS surface to existing airliner fuselage shapes (maintaining the same control point arrangement) and use the linear combination approach to populate the design space defined by these basis fuselages. We envisage this 
type of approach being particularly suited to the concise parameterisation of very complex shapes, as it is a simple way of reducing dimensionality using case-based knowledge about what constitutes a 'reasonable' shape.

\section{Acknowledgements}

We would like to thank the Royal Academy of Engineering and the Engineering and Physical Sciences Research Council (EPSRC) for the financial support of the first author's work.

\section{References}

${ }^{1}$ Jameson, A., "Aerodynamic Design via Control Theory," Journal of Scientific Computing, Vol. 3, No. 3, 1988, pp. 233-260.

${ }^{2}$ Jameson, A., "Optimum aerodynamic design using control theory," in Hafez, M. and Oshima, K.(eds), Computational Fluid Dynamics Review, John Wiley \& Sons, 1995.

${ }^{3}$ Hicks, R. M. and Henne, P. A., "Wing Design by Numerical Optimization," Journal of Aircraft, Vol. 15, 1978, pp. 407-412.

${ }^{4}$ Reuther, J., Jameson, A., Alonso, J. J., Remlinger, M. J., and Saunders, D., "Constrained Multipoint Aerodynamic Shape Optimization using an Adjoint Formulation and Parallel Computers," Journal of Aircraft, Vol. 36, No. 1, 1999, pp. 61-74.

${ }^{5}$ Kulfan, B. M., "Universal Parametric Geometry Representation Method," Journal of Aircraft, Vol. 45, No. 1, 2008, pp. $142-158$.

${ }^{6}$ Lépine, J., Guibault, F., J-Y., T., and Pépin, F., "Optimized Nonuniform Rational B-Spline Geometrical Representation for Aerodynamic Design of Wings," AIAA Journal, Vol. 39, No. 11, 2001, pp. 2033-2041.

${ }^{7}$ Painchaud-Ouellet, S., Tribes, C., Trépanier, J.-Y., and Pelletier, D., "Airfoil Shape Optimization Using a Nonuniform Rational B-Splines Parameterization Under Thickness Constraint," AIAA Journal, Vol. 44, No. 10, 2006, pp. 2170-2178.

${ }^{8}$ Sobieczky, H., "Parametric Airfoils and Wings," Notes on Numerical Fluid Mechanics, Fuji, K. and Dulikravich, G. S. (Eds.), Vol. 68, 1998, pp. 71-88.

${ }^{9}$ Robinson, G. M. and Keane, A. J., "Concise Orthogonal Representation of Supercritical Airfoils," Journal of Aircraft, Vol. 38, No. 3, 2001.

${ }^{10}$ Harris, C. D., "NASA Supercritical Airfoils; A Matrix of Family-Related Airfoils," Technical Paper 2969, NASA, 1990.

${ }^{11}$ Ferguson, J., "Multivariable Curve Interpolation," Journal of the Association for Computing Machinery, Vol. 11, No. 2, 1964, pp. 221-228.

${ }^{12}$ Sóbester, A. and Keane, A., "Airfoil Design via Cubic Splines - Ferguson's Curves Revisited," AIAA-2007-2881, 2007.

${ }^{13}$ Cox, M. G., "The Numerical Evaluation of B-splines," Journal of the Institute of Mathematics and its Applications, Vol. 10, 1972, pp. 134-149.

${ }^{14}$ De Boor, C., "On Calculating with B-splines," Journal of Approximation Theory, Vol. 6, 1972, pp. 50-62.

${ }^{15}$ Piegl, L. and Tiller, W., The NURBS Book, Springer-Verlag, Heidelberg, 1997.

${ }^{16}$ Versprille, K. J., Computer-Aided Design Applications of the Rational B-spline Approximation Form, Ph.D. thesis, Syracuse University, 1975.

${ }^{17}$ Schramm, U., Pilkey, W. D., DeVries, R. I., and Zebrowski, M. P., "Shape Design for Thin-Walled Beam Cross Sections Using Rational B-Splines," AIAA Journal, Vol. 33, No. 11, 1995, pp. 2205-2211.

${ }^{18}$ Samareh, J. A., "Novel Multidisciplinary Shape Parameterization Approach," Journal of Aircraft, Vol. 38, No. 6, 2001, pp. 1015-1024. 
${ }^{19}$ Jenkins, R. V., Hill, A. S., and Ray, E. J., "Aerodynamic performance and pressure distributions for a NASA SC(2)-0714 airfoil tested in the Langley 0.3-meter transonic cryogenic tunnel," Technical Memorandum NASA-TM-4044, NASA Langley Research Center, 1988.

${ }^{20}$ Boerstoel, J. W. and Huizing, G. H., "Transonic Shock-Free Aerofoil Design by an Analytic Hodograph Method," Journal of Aircraft, Vol. 12, No. 9, 1975, pp. 730-736.

21 "Experimental Data Base for Computer Program Assessment - Report of the Fluid Dynamics Panel Working Group 04," Advisory Report AR-138, AGARD, 1979.

${ }^{22}$ Wilby, P. G., "The Design and Aerodynamic Characteristics of the RAE 5215 Aerofoil," Current Paper 1386, Procurement Executive, Ministry of Defence, Aeronautical Research Council, 1974.

${ }^{23}$ Lagarias, J., Reeds, J. A., Wright, M. H., and Wright, P. E., "Convergence Properties of the Nelder-Mead Simplex Method in Low Dimensions," SIAM Journal of Optimization, Vol. 9, No. 1, 1998, pp. 112-147.

${ }^{24}$ Broyden, C. G., "The Convergence of a Class of Double-Rank Minimization Algorithms," Journal of the Institute of Mathematics and its Applications, Vol. 6, 1970, pp. 76-90.

${ }^{25}$ Monson, C. K. and Seppi, K. D., "Linear Equality Constraints and Homomorphous Mappings in PSO," Proceedings of the Congress on Evolutionary Computation, 2005, pp. 73-80. 